\title{
Pain and Cognition: A Narrative Review of the Effects of Chronic Pain
}

\section{(1) Forat Ferkauf Graduate}

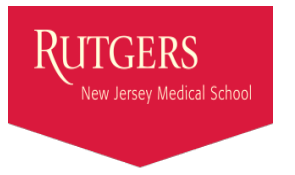

Background

\section{on Neuropsychological Functioning}

Elizabeth Lichtman and Nissan Shlomo Frager

Ferkauf Graduate School of Psychology

Rutgers New Jersey Medical School

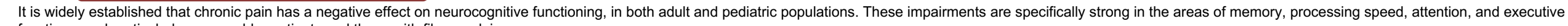
functions, and particularly among older patients and those with fibromyalgia.

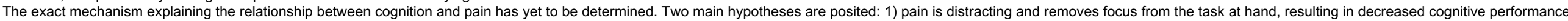
and 2) the neural systems involved in both pain and cognition are closely linked and may even modulate one another reciprocally.

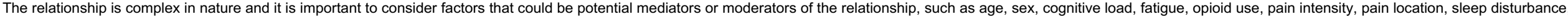

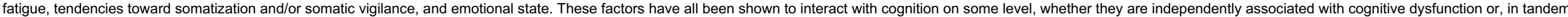
with pain, disrupt cognitive processes.

\section{Fibromyalgia}

Commonly referred to as "FibroFog", patients often report cognitive difficulties.

Research shows that patients with fibromyalgia exhibit significantly

worse performance on

neuropsychological testing than healthy controls, specifically in regard to selective attention, response inhibition, short- and long-term memory, and executive functions of set-shifting and updating.

\section{Migraine}

Deficits in attention, language use and visuospatial skills, even when controlling for other factors involved in the relationship, such as mood and medications. However, it is notable that the medication topiramate, which is used to treat chronic migraine, is ussociated with additional deficits in attention.

\section{Traumatic Brain Injury}

Research in this area is very limited, as the injury can potentially be a precipitant of both the pain and the neurocognitive impairment, making it hard to isolate these effects. However, the literature does show that veterans with TBl and chronic pain are likely to experience

\begin{tabular}{l|l}
\hline Rheumatoid Arthritis & $\begin{array}{l}\text { General chronic pain in } \\
\text { pediatric populations }\end{array}$ \\
$\begin{array}{l}\text { Deficits in attention, working } \\
\text { memory, visual and verbal } \\
\text { memory, visuospatial } \\
\text { processes, and executive } \\
\text { function }\end{array}$ & $\begin{array}{l}\text { Deficits in executive } \\
\text { functioning, specifically } \\
\text { sustained attention and } \\
\text { working memory }\end{array}$ \\
\end{tabular}

\section{Complex Regional Pain Syndrome (CRPS)}

- Patients with CRPS show distorted body representation, such as perceiving the affected limb as being larger, smaller, lighter, or heavier relative to its true size and weight. This distortion can also include a lost sense of ownership of one's limb, or incense feel

- Patients also report symptoms resembling hemispatial neglect, Which follows a brain lesion and involves attention deficits that are specific to items in one side of space. However, the performance of fully support this symptom, leading rearchers to believe it may be fully support this symplom, leading researchers to believe it may be

- Impairment of non-spatially- lateralized higher cognitive functions, such as working memory, verbal learning and memory, and nonlateralized attention, are present but not unique to CRPS, and may be explained as an effect of the pain, similar to other chronic pain conditions.

\section{Novel Treatments: Recent Research}

The following treatments have recently been shown to improve cognitive functioning chronic pain patients:

\section{- Virtual Reality (VR)}

The technological tool was used in a rehabilitative way by stimulating realistic environments and real-life exercises that were tailored to the specific needs of the patient. This technique has been shown to engage neuronal circuits which promote learning and recovery, and the results this study showed that VR-based motor training was able to improve chronic pain-related cognitive dysfunction.

\section{- Computerized cognitive training}

Computerized game-like cognitive training exercises were successful in enhancing cognitive functioning in patients with chronic pain, but more research needs to be done to determine whether the improvements wi transfer to functional and clinical outcomes.

Transcranial direct current stimulation combined with working

\section{memory training}

To enhance cognitive functions in patients with fibromyalgia, anodal transcranial direct current stimulation (tDCS) was performed over the dorsolank ding a working nent in mory relevance for top-down treatment approaches in fibromyalgia.

\section{Future Research}

- Further investigation into the role that moderators and mediators of the relationship between pain and cognition play to more efficiently target cognitive complaints

- Better understand the way chronic pain affects more complex executive skills, such as self-monitoring

- Further examine neural mechanisms and pathways common to both pain

perception and neurocognitive functioning

- More longitudinal studies to track cognitive changes over time in response to a treatment in order to better understand the effects of different pain treatments and management programs

\section{Clinical Implications}

Clinician should carefully evaluate patient with chronic pain for other factors, such as cognitive and emotional well-being.

Pain treatment should also target associated symptoms, such as low-mood, sleep issues, and cognitive impairment.

- Teachers and school psychologists should be aware of the effects of chronic pain on cognitive functioning, and multidisciplinary treatments for children and adolescents developed to incorporate cognitive training and help students achieve academic success. When assessing cognitive functioning in patients with chronic pain, the clinician should try to reduce discomfort by ensuring a try to reduce discom port by encuring a comfortable seating position and allowing
frequent breaks. The effects of the pain on fequent breaks. The effock of the pain on factors such as mood and sleep should also be results of the neuropsychological tests.

\section{References}

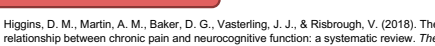

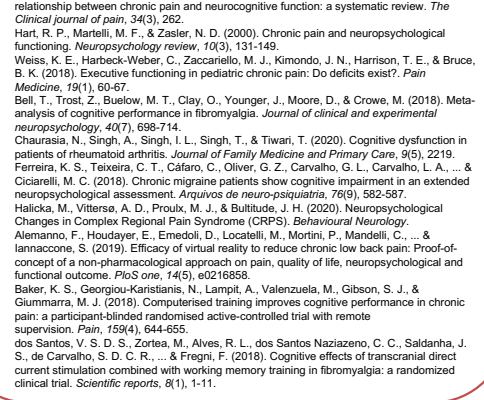

